\title{
OPINION
}

\section{Oral bacillus Calmette-Guérin vaccine against tuberculosis: why not?}

\author{
Renata Monteiro-Maia, Rosa Teixeira de Pinho/+ \\ Laboratório de Imunologia Clínica, Instituto Oswaldo Cruz-Fiocruz, Rio de Janeiro, RJ, Brasil
}

The bacillus Calmette-Guérin (BCG) vaccine is the only licensed vaccine for human use against tuberculosis (TB). Although controversy exists about its efficacy, the BCG vaccine is able to protect newborns and children against disseminated forms of TB, but fails to protect adults against active forms of TB. In the last few years, interest in the mucosal delivery route for the vaccine has been increasing owing to its increased capacity to induce protective immune responses both in the mucosal and the systemic immune compartments. Here, we show the importance of this route of vaccination in newly developed vaccines, especially for vaccines against TB.

Key words: vaccine - BCG - tuberculosis - mucosal - intradermal - oral

Vaccination is one of the most successful accomplishments of medical science and no other single intervention has had such an overwhelming effect on reducing mortality rates resulting from childhood diseases (Zaman et al. 2013). Vaccines play a central role in decreasing child mortality rates and increasing life expectancy rates. Vaccination has also resulted in the complete eradication of smallpox and in a dramatic reduction in diseases caused by several infectious agents, including bacteria and viruses (Kim et al. 2012).

The majority of vaccines used today are parenteral vaccines (Owen et al. 2013). Mucosal vaccination can be achieved via a number of routes, including oral, intranasal (IN), pulmonary, rectal or vaginal (Zaman et al. 2013). Oral mucosal vaccines have considerable advantages compared to systemic injections, including ease of administration, improved practicality for mass vaccination (i.e., not requiring trained personnel or risking contaminated needle sticks), increased patient compliance and ease of production due to a decreased need to purify bacterial by products such as endotoxin, as the gut already harbours trillions of commensal bacteria (Kim et al. 2012, Owen et al. 2013). In addition, systemic vaccines do not induce a sustained mucosal immune response. Mucosal vaccines can induce both systemic and mucosal immunity, including antigen (Ag)-specific response, especially at mucosal surfaces, which are the frontlines of pathogen infections (Ranasinghe 2014).

Mucosal surfaces are mainly represented by the gastrointestinal, respiratory and urogenital (UR) tracts and are therefore vulnerable to infection by pathogenic microorganisms (Pavot et al. 2012). They serve as gateways with

doi: 10.1590/0074-0276140091

Financial support: FIOCRUZ, INCT-TB, CNPq

+ Corresponding author: rospinho@ioc.fiocruz.br

Received 12 March 2014

Accepted 16 June 2014 a surveillance function for the acceptance of beneficial Ags from the outside environment and an immunological function for the rejection of non-beneficial Ags (Sato \& Kiyono 2012). The mucosal area maintains its integrity through coordinated interactions between the microbial flora, the physical barrier properties of the mucosa and the immune defence mechanisms (Kim et al. 2012).

The mucosal immune system consists of an integrated network of tissues, lymphoid and non-lymphoid cells and effector molecules such as antibodies, chemokines and cytokines. These host factors respond to pathogen invasion and infection (and to mucosal vaccines) by orchestrating innate and adaptive immune responses to confer protection (Woodrow et al. 2012).

Ags administered at mucosal surfaces are generally less immunogenic and tend to induce tolerance, as the host strives to maintain mucosal homeostasis by responding to mucosal Ags with a tolerant immune response (Rhee et al. 2012). Indeed, only a very limited number of mucosal vaccines have been approved for human use and are on the market: the oral polio vaccine, the oral killed-wholecell B subunit and live-attenuated cholera vaccines, the oral live-attenuated typhoid vaccine, the oral bacillus Calmette-Guérin (BCG) live vaccine [used in Brazil for vaccination against tuberculosis (TB) up until the 1970s] and the oral adenovirus vaccine (restricted to military personnel only) (Rhee et al. 2012). Efforts have revolved around developing effective mucosal vaccines and/or immunotherapies that are more efficient in delivering the appropriate Ags to the mucosal immune system. These efforts have focused on developing effective and safe mucosal adjuvants or immunoregulatory agents that provide protective immunity against infectious agents or induce the suppression of peripheral immunopathological disorders, respectively (Holmgren et al. 2003).

Mucosal vaccines, in contrast to injected vaccines, have been reported to provide additional secretory antibody-mediated protection against pathogens at the mucosal site of entry (Rhee et al. 2012). Important virtues of mucosal vaccination are their capacity to induce protective immune responses both in the mucosal and 
systemic immune compartments (Rhee et al. 2012), as well as to trigger both humoral and cell-mediated immune protection and to strongly induce long-term $\mathrm{B}$ and T cell memory responses (Lycke 2012).

Lycke (2012) affirms that protection against pathogens can be effectively achieved by directing memory and effector immune cells to the mucosal membranes through tissue-specific homing receptors. B and T cells acquire mucosal homing properties only in the draining lymph nodes specialised dendritic cells (DCs) that migrate from the mucosal tissues to these lymph nodes. Hence, vaccination via the intramuscular or subcutaneous routes poorly promotes immune protection at mucosal membranes. Following mucosal immunisation, Ag-triggered $\mathrm{B}$ and $\mathrm{T}$ cells leave the draining lymph nodes, transit through the lymph, enter the blood circulation and then "seed" the mucosal tissues (Lycke 2012).

To induce effective mucosal immune responses, a vaccine should be directed toward the main sites of mucosal immune activation. Inductive sites of the mucosal immune system include the organised lymphoid tissues such as the tonsils in the upper airways and the Peyer's patches and appendix in the intestines. These organised lymphoid tissues reside directly below the mucosal epithelium (Woodrow et al. 2012).

A prerequisite for successful mucosal vaccination is that the orally introduced Ag should be transported across the mucosal surface into the mucosa-associated lymphoid tissues (MALT). In particular, $\mathrm{M}$ cells, which are specialised epithelial cells, are responsible for Ag uptake into MALT. In addition, the rapid and effective transcytotic activity of $\mathrm{M}$ cells makes them an attractive target for mucosal vaccine delivery, although simple transport of the Ag into M cells does not guarantee the induction of specific immune responses (Kim et al. 2012).

The migration of immune cells from mucosal inductive to effector tissues is the cellular basis for the common mucosal immune system (CMIS). Mucosal vaccination elicits immune responses in distant multiple mucosal effector sites. Although it has been shown that gut-associated lymphoid tissue (GALT) and nasalassociated lymphoid tissue (NALT) share common features, it is also clear that a compartmentalisation occurs between the oral and nasal immune systems. Thus, oral immunisation mainly elicits Ag-specific immune responses in the small intestine, in the proximal part of the large intestine and in mammary and salivary glands, whereas nasal immunisation induces mucosal immunity in the UR tract, the nasal and oral cavities and the cervicovaginal mucosa (Fujkuyama et al. 2012). Nasal and sublingual immunisation can induce immune responses in the genital tract (Holmgren \& Svennerholm 2012).

Mucosal inductive sites, including GALT and NALT, collectively comprise a MALT network for the provision of a continuous source of memory B and T cells to mucosal effector sites. MALT contains T-cell zones, B cellenriched areas containing a high frequency of surface $\operatorname{IgA}(\operatorname{sg} \mathrm{A})$-positive B cells and a subepithelial area with argon plasma coagulation (APCs) for the initiation of specific immune responses. MALT is covered by a follicleassociated epithelium that consists of a subset of differen- tiated microfold (M) epithelial cells, columnar epithelial cells and lymphoid cells, all of which play a central role in the initiation of mucosal immune responses. M cells take up Ags (Ags) from the lumen of the intestinal and nasal mucosa and transport them to the underlying APCs, including DCs. In addition, recent studies have identified isolated lymphoid follicles (ILFs) in the mouse small intestine. The ILFs have been identified as part of GALT and, as such, are a mucosal inductive tissue. These ILFs mainly contain B cells, DCs and M cells in the overlying epithelium. In addition, most recent studies showed that tear duct-associated lymphoid tissue and conjunctivaassociated lymphoid tissue play a role as mucosal inductive tissues. Mucosal effector sites, including the lamina propria regions of the GI, the upper respiratory and the reproductive tracts, secretory glandular tissues and intestinal intraepithelial lymphocytes, contain Ag-specific mucosal effector cells such as IgA-producing plasma cells and B and T cells (Fujkuyama et al. 2012).

One of the most important infectious diseases that use the mucosa as an entry gateway is TB. This disease is caused by a highly robust bacterial pathogen, Mycobacterium tuberculosis $(M t b)$, which resists and even subverts protective immunity. TB currently afflicts approximately nine million individuals (Kaufmann 2013). Its successful survival strategy is reflected by the epidemiology of the disease. TB remains a major global health problem. In 2012, an estimated 8.6 million people developed TB and 1.3 million died from the disease [including 320,000 deaths among human immunodeficiency virus (HIV)positive people]. The number of TB deaths is unacceptably large given that most of these deaths are preventable (WHO 2013). This situation is worsened, especially in poorer countries, where TB coincides with immunocompromised HIV-infected individuals and where latent TB infection (LTBI) and multidrug resistance are major contributing factors to the increased burden of disease (Clark et al. 2010). The treatment of TB is achieved through the use antibiotic therapy and prevention is through vaccination with Mycobacterium bovis BCG.

$\mathrm{BCG}$, the vaccine most widely used against $\mathrm{TB}$ worldwide, is derived from $M$. bovis and has been attenuated after 230 passages over a period of 13 years. Since its attenuation, the original BCG strain has produced many descendant strains that have been distributed and used in many countries and regions around the world (Zhang et al. 2013).

This attenuation promoted genomic deletions, that together with the evolution of $M$. bovis, resulted in 16 genomic regions of differentiation (RD) (RD1-RD16, plus $n R D 18$ ), when compared with the $M t b$ genome (Costa et al. 2014). In additions, a series of genetic modifications, such as deletions and insertions, have occurred that currently define several groups of the BCG substrains. The “early" BCG, Group 1 (1921-1925; BCG Moscow, BCG Moreau and BCG Tokyo), which were distributed first by Calmette and are still in use today, seem to be the closest genetically to the original strain. In the $1920 \mathrm{~s}$, loss of a DNA sequence upstream of the important regulator gene $p h o P$ gave rise to Group 2 BCG Sweden and BCG Birkhaug. After 1931, Group 3 emerged, which includes 
BCG Glaxo and BCG Copenhagen, currently manufactured by Staten Serum Institute, Denmark, and sold as BCG Danish 1331. A "late" Group 4 includes BCG Tice (1934) and BCG Connaught (1948), both of which are no longer used for vaccination, but are the principal substrains used for bladder cancer immunotherapy in Europe and the United States of America (Brosch et al. 2007, Gan et al. 2013).

BCG Moreau, the strain used in Brazil, has a unique characteristic that corresponds to a 7,608 bp deletion (RD16) compared to the Mtb genome. Gomes et al. (2011) also confirmed the presence of tandem duplication DU2-I and a Moreau-specific deletion in fadD26ppsA (976 bp). RD16 is a $7.6 \mathrm{~kb}$ DNA section encoding Rv3405 that is responsible for colony morphology characteristics and for the formation of cell membrane constituents (Honda et al. 2006). Studies have shown that "early" derived strains, such as the BCG Moreau, are more immunogenic and may confer better protection against TB (Gomes et al. 2011).

The BCG vaccine, the only vaccine licensed against $\mathrm{TB}$, is one of the most widely used vaccines because it is both inexpensive and safe (Kashyap et al. 2010). It is effective in preventing the most severe disseminated forms of the disease in children and newborns (Clark et al. 2010), but it fails to protect against adult pulmonary TB (Kaufmann 2010). The BCG vaccine is contraindicated in infants infected with HIV (Hawkridge 2009).

Meta-analysis studies have confirmed that BCG protects children, providing $>80 \%$ efficacy against severe forms of $\mathrm{TB}$, including tuberculous meningitis and miliary TB. In contrast, evidence for protection against pulmonary TB in adolescents and adults remains contentious, as efficacy estimated from clinical trials, observational case control studies and contact studies range from $0-80 \%$ (Liu et al. 2009).

The reasons for variable BCG efficacy are unknown, but it is hypothesised that a number of factors may contribute to the variability. These factors include differences among vaccine strains used, pre-exposure of populations to environmental mycobacteria, genetic or nutritional differences among human populations and differences among clinical strains of Mtb (Clark et al. 2010).

The protective efficacy of BCG also depends on the geographical location, as BCG efficacy has been shown to be reduced in populations that live in rural areas closer to the Equator (Burl et al. 2010). In a recent review, Mangtani et al. (2014) described a well-established association between protection and geographic location.

Despite the relative efficacy of BCG in infants, one of the major unanswered questions is why the BCG vaccine fails to prevent pulmonary TB in adolescents. It has been proposed that immune memory wanes in adolescence, which is the most critical period for TB infection and/or its progression to active disease (Ottenhoff $\&$ Kaufmann 2012). One possible explanation is the fact that immunological memory is induced by BCG at an early age (neonates or infants) when the immune system is not yet fully mature. However, other factors may also contribute to decreasing BCG efficacy and/or enhanced susceptibility of young adults to TB.
There are genetic differences between BCG vaccines that suggest that the BCG strains used have evolved since 1921. Brosch et al. (2007) used genome sequencing to postulate that BCG vaccines derived before 1930 or 1940 may be immunologically superior to the more recent and widely used variants. Mangtani et al. (2014) found little evidence for an association between the estimated effects of BCG and the year each trial commenced or that effects varied according to the groups proposed. Those BCG groups include strains currently in use: Denmark (in DU2 Group III), Russia (in DU2 Group I) and Japan (also in DU2 Group I).

It is important to remember that other proposed explanations include human genetic differences, genotypic differences between infecting mycobacteria and a variety of proposed explanations for the association of protection with geographic latitude: exposure to ultraviolet light (due to its mycobacterial killing effect), levels of vitamin $\mathrm{D}$, helminthic infestation or the effect of poor nutrition on immune responses (Mangtani et al. 2014).

Another important aspect is the impact of non-tuberculous mycobacteria (NTM) infection on BCG vaccination. There is some evidence that prior exposure to NTM may affect the efficacy of BCG vaccines. This possible interference may be the cause of the reduced efficacy of the BCG vaccine demonstrated in the Chingelput BCG trial and it may explain the geographic differences in vaccine efficacy. In fact, repeated exposure to NTM in tropical regions is believed to be the main explanation for the low efficacy of BCG vaccines in these areas. Furthermore, because of cross-reactivity among mycobacterial species, exposure to NTM may provide some protection against $\mathrm{TB}$ and it can also alter the results of purified protein derivative (PPD) skin tests (Valadas 2004).

Due to the controversy over the effectiveness of the BCG vaccine, many studies researching new vaccine strategies have been developed. Increases in investments over the last years have led to advances in the development of new TB vaccines, diagnostic methods and drugs. Although several TB vaccines are in Phase 2 and $2 \mathrm{~b}$ trials, vaccine evaluation is a lengthy and high-risk process (Gröschel et al. 2014).

Over 10 candidate $\mathrm{TB}$ vaccines designed either to boost the BCG vaccine or replace it are at different stages of clinical testing (Tameris et al. 2013). The majority of preventive vaccines build on immunity induced following priming with BCG. These booster vaccines are either viral vectors expressing one or more Mtb Ags or protein-adjuvant formulations comprising fusion proteins of up to four Mtb Ags (Weiner 3rd \& Kaufmann 2014). TB vaccines can be administered either pre-infection, designed to prevent infection from occurring or post-infection, designed to prevent latent infection progression (Esmail et al. 2014).

Strategies used on new vaccines against TB development include subunit vaccines, production of non-recombinant viral vectors and recombinant BCG (rBCG) construction. The construction of $\mathrm{rBCG}$ includes overexpression of $M t b$ immunodominant Ags expressed by $\mathrm{BCG}$, insertion of $M t b$ immunodominant Ags absent on 
BCG (overexpression with reintroduction of genes lost during BCG attenuation) and BCG modification to induce $\mathrm{CD}^{+} \mathrm{T}$ cell-specific immune response proteins and cytokines (Costa et al. 2014).

The BCG vaccine was developed by Calmette and Guérin as an oral vaccine. In Brazil, Assis (1950) demonstrated that repeated oral doses of BCG Moreau were highly effective in preventing TB. Brazil routinely employed single-dose oral immunisation with $100 \mathrm{mg}$ of BCG Moreau up to the mid-1970s (Cosgrove et al. 2006).

In 1921, Calmette chose the oral route for BCG vaccination for its simplicity of administration, its penetration through the intestinal epithelium in newborn animals and babies and for its capacity to induce specific mycobacterial immunity through this route. More recently, it has been shown that BCG is able to cross the intestinal barrier through the $M$ cells of Peyer's patches. In fact, BCG was found in the Peyer's patches of mice $6 \mathrm{~h}$ postadministration (Lagranderie et al. 2000).

During the years 1924-1926, at Ulleval Hospital in Oslo (Norway), it was observed that oral administration of BCG produced no "allergic skin" response, one of the criteria accepted at the time as evidence of immunity against TB; it was thus decided that a switch to a parenteral vaccine (subcutaneously) was needed. The results showed that parenteral administration led to an "allergic reaction" to tuberculin or the tuberculin skin test (TST) (Heimbeck 1948) . Consequently, the parenteral route became popular in the Nordic countries, especially after 1927, when Walgreen improved vaccination through the intradermal route and inoculation by using $0.1 \mathrm{mg}$ of BCG in individuals of any age with a negative skin test (Benévolo-de-Andrade et al. 2005).

In 1930, there was a serious accident in Lübeck (Germany) which caused profound changes in BCG vaccination, reinforcing the change of route. According to Benévolo-de-Andrade (2005), 250 children were supposedly vaccinated with BCG and 73 died from TB in the first year, while another 135 developed signs and symptoms of disease. Subsequent investigations revealed that a culture of $M t b$, isolated from a sick child, was kept in the same incubator with the BCG and, during the vaccine preparation, the vaccine became contaminated and contained $1 / 3$ of BCG and $2 / 3$ of the tuberculous bacillus. Based on this, oral vaccination was replaced, in most countries, by the intradermal route (Gheorghiu 1996). Brazil maintained the use of the oral vaccine until the mid-seventies, when it was replaced by the intradermal route. This change in the route of immunisation in Brazil was mainly for medical pressure based on poor skin responses of individuals immunised orally (Succi 1985). After the Second World War, the use of BCG increased in Europe and in developing countries (Succi 1985).

By the beginning of the 1940s, other routes were evaluated because: (i) oral delivery of BCG was sometimes causing cervical adenitis and (ii) high doses of oral BCG were required to induce positive delayed-type-hypersensitivity (DTH) response to tuberculin. Today it is established, both in animal models and in humans, that BCG-induced DTH does not correlate with protection because T-cell subsets and recognised Ags involved in DTH differ from those inducing protection (Badell et al.
2009). No cervical adenitis cases were reported in Brazil, where up to $200 \mathrm{mg}$ of BCG Moreau wild type was administered per os to newborns between 1945-1977 (Badell et al. 2009).

BCG-based vaccines can potentially provide a safe and effective tool to mimic natural infection and stimulate both innate and acquired immunity under relatively "natural" conditions of gut infection (Schreiber et al. 2010).

Monteiro-Maia et al. (2006) observed that two individuals who received oral BCG vaccine boosts showed an alteration in their humoral immune response, measured as an isotype shift from IgG to IgA, suggesting that oral revaccination is capable of provoking cellular and humoral responses. This response was independent of the route used in previous vaccination. Given that TB affects an important mucosal site, the respiratory tract, the potential use of oral booster vaccination in immunisation programs is of interest. Subjects who were not boosted were not capable of mounting this shift in immunoglobulin isotype for the Ags tested. Hoft et al. (2000) proposed a combination of oral and intradermal routes for BCG vaccination with the objective of inducing protective mucosal and systemic immunity against initial infection and systemic progression.

Cosgrove et al. (2006) reported that oral-delivery of BCG Moreau to humans could elevate interferon (IFN)- $\gamma$ responses among subjects who had received a prior (parenteral-route) BCG immunisation. This indicates that an orally delivered BCG may have the potential to act as either a primary vaccine or as a boosting agent.

Researchers over the last several decades have tried to determine how our immune system fights $M t b$ infection. Previous evidence shows that the development of either a $T_{H} 1$ or a $T_{H} 2$ response during mycobacterial infection can lead to different clinical outcomes. $\mathrm{T}_{\mathrm{H}} 1$ cytokines stimulate cell-mediated immunity (CMI) and anti-intracellular pathogen responses, while $\mathrm{T}_{\mathrm{H}} 2$ cytokines stimulate predominantly anti-extracellular pathogen humoral responses and are associated with progressive infection of $M t b$ (Ordway et al. 2005). It is known that $\mathrm{CD} 4^{+} \mathrm{T}$ cells and the pro-inflammatory cytokine IFN- $\gamma$ are required to control $M t b$ infection in humans and in mice (Thakur et al. 2012). Other aspects of the immune response shown to play a role in protection against TB include $\mathrm{CD} 8^{+} \mathrm{T}$ cells, $\mathrm{T}_{\mathrm{H}} 17$ cells, $\gamma \delta \mathrm{T}$ cells, CD1-restricted invariant natural killer $\mathrm{T}$ cells and mucosal-associated invariant $\mathrm{T}$ cells (Khader et al. 2007, Sada-Ovalle et al. 2008, Gold et al. 2010, Meraviglia et al. 2011, Bold \& Ernst 2012).

According to Thakur et al. (2012), IFN- $\gamma$ is essential for the defence against $M t b$ infection. This was revealed by experimental studies using knockout mice and through the observation of unusually severe mycobacterial infections in patients with defects in either the IFN- $\gamma$ or the interleukin (IL)-12 signalling pathways. The role of $\mathrm{CD}^{+} \mathrm{T}$ cells in the defence against $M t b$ infection has been inferred from the increased reactivation of latent $M t b$ infections in $\mathrm{CD}^{+} \mathrm{T}$ cell deficient patients following HIV infection and from severe TB observed in $\mathrm{CD} 4^{+}$ $\mathrm{T}$ cell-deficient mice. These clinical and experimental findings have led to a widely accepted model positing that the critical immunologic mechanism of anti-myco- 
bacterial immunity involves $\mathrm{CD} 4^{+} \mathrm{T}$ cells that secrete IFN- $\gamma$ to activate bactericidal functions of $M t b$-infected macrophages. Substantial evidence indicates that IFN- $\gamma$ can activate murine macrophages to limit $M t b$ growth, but the relative importance of this bactericidal mechanism and the cellular sources of IFN- $\gamma$ are unknown. Evidence for a $\mathrm{CD}^{+} \mathrm{T}$ cell-dependent, IFN- $\gamma$-independent mechanism of killing has been suggested by the finding that the frequency of $M t b$-specific, IFN- $\gamma$-producing cells following immunisation do not correlate with protection against infection and that depletion of $\mathrm{CD}^{+} \mathrm{T}$ cells exacerbates $M t b$ infection in mice, despite the ongoing expression of IFN- $\gamma$ (Thakur et al. 2012).

During $M t b$ infection, major histocompatibility complex (MHC) class II and class I restricted $\mathrm{CD} 4^{+}$ and $\mathrm{CD} 8^{+}$, CD1-restricted and $\gamma \delta \mathrm{T}$ lymphocytes are activated by Ags through MHC class II and I molecules, lipid Ags through CD1 molecules and phospholigands through $\gamma \delta \mathrm{T}$ cells, respectively. Activated T lymphocytes release IFN- $\gamma$ and other cytokines which will in turn activate macrophages to eliminate bacteria. $\mathrm{CD}^{+}$ $\mathrm{T}$ lymphocytes are differentiated into several different effector cells such as $T_{H} 1, T_{H} 2, T_{H} 17$ and regulatory $T$ cells (Treg). $\mathrm{T}_{\mathrm{H}} 1$ cells mainly produce IFN- $\gamma$ controlling intracellular infection including $M t b$, whereas $\mathrm{T}_{\mathrm{H}} 2$ cells produce IL-4, IL-5 and IL-13 mediating humoral immunity. Treg cells produce IL-10, negatively regulating both IFN- $\gamma$ and IL-17 responses (Li et al. 2012).

According to Ling et al. (2013), IL-17A is required to induce the formation of mature granuloma after $M t b$ infection. Mice deficient in IL-17A exhibit impaired granuloma formation and weakened protective immunity against $M t b$ infection. Furthermore, IL-17A promotes the production of chemokines in mice during $M t b$ challenge, leading to the recruitment of neutrophils and IFN- $\gamma$-producing $\mathrm{CD}^{+} \mathrm{T}$ cells, which subsequently contribute to the restriction of $M t b$ growth in the lungs.

Efficient control of $M t b$ infection mainly depends on interactions between infected macrophages and DCs with Ag-specific T-lymphocytes secreting IFN- $\gamma$. Activation by IFN- $\gamma$ confers tuberculostatic and tuberculocidal capacities upon macrophages, reflected by mycobacterial outgrowth in mice and humans with defects in IFN- $\gamma$ signalling. IFN- $\gamma$-induced factors involved in the protection against TB in vivo include inducible nitric oxide synthase, which generates reactive nitrogen intermediates and the small GTPase LRG-47. As a result of T$\mathrm{CMI}$, replication of $M t b$ is confined; however, a residual number of mycobacteria may enter a dormant stage such that latently infected individuals stand a $10 \%$ risk of disease reactivation later in life (Kahnert et al. 2006).

Testing cellular immune reactivity to Mtb Ags is a common diagnostic procedure when suspecting an infection with $M t b$. Without clinical, radiological or microbiological proof of the active disease, the immune reactivity to $M t b$ Ags is termed LTBI. The traditional intracutaneous tuberculin test (Mendel Mantoux test) requires the patients to be seen twice, is prone to false positive results after BCG vaccination and to false negative reaction in states of immunosuppression. The in vitro IFN $-\gamma$ release assays provide an enhanced speci- ficity after BCG vaccination and infection with non-TB mycobacteria, as well as the convenience of a onetime blood test (Felber \& Graninger 2013).

BCG strain Moreau Rio de Janeiro (MRDJ) had been continually used as an oral vaccine in the national programme in Brazil until 1974 and remained commercially available until 2005 (Ho et al. 2010). The BCG Moreau strain still remains available for human oral administration and has a good safety record with fewer reported side effects compared to other BCG strains (Clark et al. 2010).

There is general agreement that effective mucosal vaccines could dramatically contribute to the improvement of global health by stimulating protective immune responses not only against mucosal infections, but also against HIV, $M t b$ and many other infections (Lycke 2012).

Oral administration could have many advantages over parenteral-route BCG immunisation, including avoidance of needles, ease of administration and low cost. Recently, oral-delivery of BCG was reported as an effective boosting vaccine to pre-existing parenteral-route BCG immunisation, using the BCG MRDJ (Vipond et al. 2008).

Oral administration has been largely superseded by intradermal administration in public health, although there is now renewed interest in oral-route delivery of BCG vaccines (Vipond et al. 2008). Mucosal BCG administration inhibited DTH responses to PPD, but induced mycobacteria-specific IFN- $\gamma$ responses in vaccinated individuals (Hoft et al. 2000).

Oral route BCG vaccines have the potential for boosting mucosal immunity in BCG-primed vaccinated animals extending the longevity of protection against TB (Vipond et al. 2008).

Oral BCG was used in recent clinical studies and it was found that revaccination orally induces circulating cellmediated immune responses, but does not induce a positive TST in responsive individuals. It is also able to induce modulation in humoral immunological responses (switch from IgG to IgA isotypes) (Monteiro-Maia et al. 2006).

Mice orally vaccinated with live $M$. bovis BCG in lipid-formulation exhibit an IFN- $\gamma$ response that can be measured systemically and the vaccine conferred protection against an aerosolised mycobacterial challenge. A single oral immunisation with lipid-formulated live BCG invoked secreted and cellular IFN- $\gamma$ responses in mice eight weeks post-vaccination, the magnitudes of which were significantly elevated in mice receiving multiple immunisations over the eight-week period. Interestingly, the magnitude of IFN- $\gamma$ responses in mice was amplified by repeated oral immunisations of live BCG, whereas the magnitude of IL-2 production did not increase with multiple immunisations (Cross et al. 2008).

Wang et al. (2010) indicated that oral vaccination with the liposomal-pcDNA $3.1^{+} / \mathrm{Ag} 85 \mathrm{~A}$ DNA is able to induce Ag-specific mucosal cellular and humoral immune responses. Orally administered liposomal-pcDNA3.1 $1^{+}$Ag85A DNA was efficiently incorporated into the mucosal epithelium of the small intestine Peyer' patches and initiated Ag85A-specific $\mathrm{T}_{\mathrm{H}} 1$ dominant immune responses, evidenced by the increased secretion of IL-2, IFN- $\gamma$ and no changes of IL-4. This enhanced $\mathrm{T}_{\mathrm{H}} 1$ dominant activation facilitated the augmentation of 
Ag-specific cytolytic activity of intestinal intraepithelial lymphocytes. Increased expression of FasL in IELs suggested that FasL-Fas pathway was closely involved into the augmented Ag-specific cytolytic activity of intestinal intraepithelial lymphocytes. Meanwhile, intestinal intraepithelial lymphocyte-derived IL-10 and transforming growth factor- $\beta$ cytokines could harness the class switching of $\operatorname{IgM}^{+} \mathrm{B}$ cells to IgA producing B cells, elevates the production of $\operatorname{sg} \mathrm{A}$ in humoral immunity which contribute greatly to the protection against bacteria in the local mucosal immunity. These data indicated that oral vaccination with the liposomal-pcDNA $3.1^{+} / \mathrm{Ag} 85 \mathrm{~A}$ DNA is able to induce Ag-specific mucosal cellular and humoral immune responses. Especially cellular compartment in the epithelium of the small intestine plays a key role in the mediation of immune responses that eliminate TB.

Cross et al. (2008) had concluded that the most appropriate mycobacterial agent to invoke a CMI response via the oral route is live BCG. When incorporated into an edible lipid matrix and delivered as a voluntary consumption vaccine, live bacilli are able to access and replicate within the alimentary tract of lymphatic tissues. This in turn promotes strong IFN- $\gamma$ responses in particular (which prior work has shown is mainly due to activated $\mathrm{CD} 4^{+} / \mathrm{CD} 44^{\mathrm{hi}} / \mathrm{CD} 62 \mathrm{~L}^{\text {lo }}$ cell populations) and can be augmented by repeated dosing of the vaccine.

Heterologous prime/boost vaccination strategies induce robust $\mathrm{T}$ cell responses and may improve protection when compared to BCG alone. Therefore, many new TB vaccine approaches under development focus on booster vaccines to enhance and extend immunity acquired after primary BCG immunisation. Regional immunity in the lung may be important for enhanced protection at the site of initial infection and IN or other mucosal-delivered vaccines might induce $M t b$ specific mucosal immunity capable of preventing TB infection (Blazevic et al. 2014).

The importance of the role of T-cells in the immune response to TB is known, but the role for B-cells in mycobacteria specific immunity cannot be ruled out. Sebina et al. (2012) determined the presence and frequencies of mycobacteria-specific memory B-cells (MBCs) in peripheral blood from clinically healthy, BCG-vaccinated and unvaccinated donors. They demonstrate that mycobacteria-specific MBCs responses are elicited after BCG vaccination, readily detected in peripheral blood and are long lived. These data combined suggested a role for B-cells in immune responses to BCG and indicated that $\mathrm{BCG}$ vaccination induces long-lived MBC responses. Similar patterns of responses were seen when we examined mycobacteria-specific antibody and T-cell responses in the donors. The data show that BCG vaccination elicits long-lived mycobacteria-specific MBC responses in healthy individuals, suggesting a more substantial role for B-cells in the response to BCG and other mycobacterial infections.

Mucosal vaccines are advantageous when compared to systemic vaccines, as they are easier to produce, easier to administer and do not involve a risk for spreading blood-borne infections. It has been demonstrated that mucosal vaccination can induce both memory $\operatorname{Ig} \mathrm{A}^{+}$and memory $\mathrm{IgG}^{+} \mathrm{B}$ cells and there is general agreement that effective mucosal vaccines are able to induce protective immune responses against $M t b$ and many other infections. Therefore, further discussion is needed to evaluate the role of this route in the immunisation against TB or in combination with the intradermal route in order to generate a more efficient and effective vaccine against TB.

\section{ACKNOWLEDGEMENTS}

To Dra Marcia de Berredo Pinho Moreira, for review the paper.

\section{REFERENCES}

Assis A 1950. Novas perspectivas da vacinação contra a tuberculose pelo BCG. O Hospital XXXVIII: 337-353

Badell E, Nicolle F, Clark S, Majlessi L, Boudou F, Martino A, Castello-Branco L, Leclerc L, Lewis DJM, Marsh PD, Gicquel B, Winter N 2009. Protection against tuberculosis induced by oral prime with Mycobacterium bovis BCG and intranasal subunit boost based on the vaccine candidate Ag85B-ESAT-6 does not correlate with circulating IFN- $\gamma$ producing T-cells. Vaccine 27: 28-37.

Benévolo-de-Andrade TC, Monteiro-Maia R, Cosgrove C, CastelloBranco LRR 2005. BCG Moreau Rio de Janeiro - An oral vaccine against tuberculosis - Review. Mem Inst Oswaldo Cruz 100: $459-465$

Blazevic A, Eickhoff CS, Stanley J, Buller MR, Schriewer J, Kettleson EM, Hoft DF 2014. Investigations of TB vaccine-induced mucosal protection in mice. Microbes Infect 16: 73-79.

Bold TD, Ernst JD 2012. CD4 ${ }^{+}$T cell-dependent IFN- $\gamma$ production by $\mathrm{CD}^{+}$effector T cells in Mycobacterium tuberculosis infection. $J$ Immunol 189: 2530-2536.

Brosch R, Gordon SV, Garnier T, Eiglmeier K, Frigui W, Valenti P, dos Santos S, Duthoy S, Lacroix C, Garcia-Pelayo C, Inwald JK, Golby P, Garcia JN, Hewinson RG, Behr MA, Quail MA, Churcher C, Barrell BG, Parkhill J, Cole ST 2007. Genome plasticity of BCG and impact on vaccine efficacy. PNAS 104: 5596-5601.

Burl S, Adetifa UJ, Cox M, Touray E, Ota MO, Marchant A, Whittle H, McShane H, Rowland-Jones SL, Flanagan KL 2010. Delaying bacillus Calmette-Guérin vaccination from birth to 4 1/2 months of age reduces post-vaccination $\mathrm{T}_{\mathrm{H}} 1$ and $\mathrm{IL}-17$ responses but leads to comparable mycobacterial responses at 9 months of age. J Immunol 185: 2620-2628.

Clark SO, Kelly DL, Badell E, Castello-Branco LR, Aldwell F, Winter N, Lewis D, Marsh PD 2010. Oral delivery of BCG Moreau Rio de Janeiro gives equivalent protection against tuberculosis but with reduced pathology compared to parenteral BCG Danish vaccination. Vaccine 28: 7109-7116.

Cosgrove CA, Castello-Branco LR, Hussell T, Sexton A, Giemza R, Phillips R, Williams A, Griffin GE, Dougan G, Lewis DJ 2006. Boosting of cellular immunity against Mycobacterium tuberculosis and modulation of skin cytokine responses in healthy human volunteers by Mycobacterium bovis BCG substrain Moreau Rio de Janeiro oral vaccine. Infect Immun 74: 2449-2452.

Costa AC, Nogueira SV, Kipnis A, Kipnis APJ 2014. Recombinant CG: innovations on an old vaccine. Scope of BCG strains and strategies to improve long-lasting memory. Front Immunol 5: 152.

Cross ML, Lambeth MR, Aldwell FE 2008. Murine cytokine responses following multiple oral immunizations using lipid-formulated mycobacterial antigens. Immunol Cell Biol 86: 214-217.

Esmail H, Barry CE, Young DB, Wilkinson RJ 2014. The ongoing challenge of latent tuberculosis. Philos Trans $R$ Soc Lond B Biol Sci 369: 1-14.

Felber A, Graninger W 2013. Weakly positive tests and chronologic variation of the QuantiFERON assay: a retrospective appraisal of usefulness. Tuberculosis (Edinb) 93: 647-653. 
Fujkuyama Y, Tokuhara D, Kataoka K, Gilbert RS, McGhee JR, Yuki Y, Kiyono H, Fujihashi K 2012. Novel vaccine development strategies for inducing mucosal immunity. Expert Rev Vaccines 11: 367-379.

Gan C, Mostafid H, Kha MS, Lewis DJM 2013. BCG immunotherapy for bladder cancer - the effects of substrain differences. Nat Rev Urol 10: 580-588.

Gheorghiu M 1996. Le BCG, vaccin contre la tuberculose: leçons du passé pour aujourd'hui. In AM Moulin, L'Aventure de la vaccination, Fayard, Paris, p. 219-228.

Gold MC, Cerri S, Smyk-Pearson S, Cansler ME, Vogt TM, Delepine J, Winata E, Swarbrick GM, Chua WJ, Yu YY, Lantz O, Cook MS, Null MD, Jacoby DB, Harriff MJ, Lewinsohn DA, Hansen TH, Lewinsohn DM 2010. Human mucosal associated invariant T cells detect bacterially infected cells. PLoS Biol 8: e1000407.

Gomes LHF, Otto TD, Vasconcellos EA, Ferrão PM, Maia RM, Moreira AS, Ferreira MA, Castello-Branco LRR, Degrave WM, Mendonça-Lima L 2011. Genome sequence of Mycobacterium bovis BCG Moreau, the Brazilian vaccine strain against tuberculosis. J Bacteriol 193: 5600-5601.

Gröschel MI, Prabowo SA, Cardona PJ, Stanford JL, Van der Werf TS 2014. Therapeutic vaccines for tuberculosis - a systematic review. Vaccine 32: 3162-3168.

Hawkridge A 2009. Clinical studies of TB vaccines. Hum Vaccin 5: 773-776.

Heimbeck J 1948. BCG vaccination of nurses. Tubercle April: 84-88.

Ho MM, Southern J, Kang HN, Knezevic I 2010. WHO informal consultation on standardization and evaluation of BCG vaccines Geneva, Switzerland 22-23 September 2009. Vaccine 28: 6945-6950.

Hoft DF, Brown RM, Belshe RB 2000. Mucosal bacille Calmette-Guérin vaccination of humans inhibits delayed-type hypersensitivity to purified protein derivative, but induces mycobacteria-specific interferon-gamma responses. Clin Infect Dis 30 (Suppl. 3): S217-S222.

Holmgren J, Czerkinsky C, Eriksson K, Mharandi A 2003. Mucosal immunisation and adjuvants: a brief overview of recent advances and challenges. Vaccine 21 (Suppl. 2): S2/89-S2/95.

Holmgren J, Svennerholm AM 2012. Vaccines against mucosal infections. Curr Opin Immunol 24: 343-353.

Honda I, Seki M, Ikeda N, Yamamoto S, Yano I, Koyama A, Toida I 2006. Identification of two subpopulations of bacillus CalmetteGuérin (BCG) Tokyo 172 substrain with different RD16 regions. Vaccine 24: 4969-4974.

Kahnert A, Seiler P, Stein M, Bandermann S, Hahnke K, Mollenkopf H, Kaufmann SH 2006. Alternative activation deprives macrophages of a coordinated defense program to Mycobacterium tuberculosis. Eur J Immunol 36: 631-647.

Kashyap RS, Husain AA, Morey SH, Panchbhai MS, Deshpande PS, Purohit HJ, Taori GM, Daginawala HF 2010. Assessment of immune response to repeat stimulation with $\mathrm{BCG}$ vaccine using in vitro PBMC model. J Immune Based Ther Vaccines 8: 3.

Kaufmann SH 2010. Novel tuberculosis vaccination strategies based on understanding the immune response. J Intern Med 267: 337-353.

Kaufmann SH 2013. Tuberculosis vaccines: time to think about the next generation. Semin Immunol 25: 172-181.

Khader SA, Bell GK, Pearl JE, Fountain JJ, Rangel-Moreno J, Cilley GE, Shen F, Eaton SM, Gaffen SL, Swain SL, Locksley RM, Haynes L, Randall TD, Cooper AM 2007. IL-23 and IL-17 in the establishment of protective pulmonary $\mathrm{CD}^{+} \mathrm{T}$ cell responses after vaccination and during Mycobacterium tuberculosis challenge. Nat Immunol 8: 369-377.
Kim SH, Lee KY, Jang YS 2012. Mucosal immune system and M cell-targeting strategies for oral mucosal vaccination. Immune Netw 12: 165-175.

Lagranderie M, Chavarot P, Balazuc AM, Marchal G 2000. Immunogenicity and protective capacity of Mycobacterium bovis BCG after oral or intragastric administration in mice. Vaccine 18: 1186-1195.

Li Q, Li J, Tian J, Zhu B, Zhang Y, Yang K, Ling Y, Hu Y 2012. IL-17 and IFN- $\gamma$ production in peripheral blood following BCG vaccination and Mycobacterium tuberculosis infection in human. Eur Rev Med Pharmacol Sci 16: 2029-2036.

Ling WL, Wang LJ, Pong JCH, Lau ASY, Li JCB 2013. A role for interleukin-17A in modulating intracellular survival of Mycobacterium bovis bacillus Calmette-Guérin in murine macrophages. Immunology 140: 323-334.

Liu J, Tran V, Leung AS, Alexander DC, Zhu B 2009. BCG vaccines. Their mechanisms of attenuation and impact on safety and protective efficacy. Hum Vaccin 5: 70-78.

Lycke N 2012. Recent progress in mucosal vaccine development: potential and limitations. Nat Rev Immunol 12: 592-605.

Mangtani P, Abubakar I, Ariti C, Beynon R, Pimpin L, Fine PEM, Rodrigues LC, Smith PG, Lipman M, Whiting PF, Sterne JA 2014. Protection by BCG vaccine against tuberculosis: a systematic review of randomized controlled trials. Clin Infect Dis 58: 470-480.

Meraviglia S, El Daker S, Dieli F, Martini F, Martino A 2011. $\gamma \delta \mathrm{T}$ cells cross-link innate and adaptive immunity in Mycobacterium tuberculosis infection. Clin Dev Immunol 2011: 11.

Monteiro-Maia R, Ortigão-de-Sampaio MB, Pinho RT, Castello-Branco LR 2006. Modulation of humoral immune response to oral BCG vaccination by Mycobacterium bovis BCG Moreau Rio de Janeiro (RDJ) in healthy adults. J Immune Based Ther Vaccines 6: 4.

Ordway DJ, Martins MS, Costa LM, Freire MS, Arroz MJ, Dockrell HM, Ventura FA 2005. Increased IL-4 production in response to virulent Mycobacterium tuberculosis in tuberculosis patients with advanced disease. Acta Med Port 18: 27-36.

Ottenhoff THM, Kaufmann SHE 2012. Vaccines against tuberculosis: where are we and where do we need to go? PLoS Pathog 8: e1002607.

Owen JL, Sahay B, Mohamadzadeh M 2013. New generation of oral mucosal vaccines targeting dendritic cells. Curr Opin Chem Biol 17: 918-924.

Pavot V, Rochereau N, Genin C, Verrier B, Paul S 2012. New insights in mucosal vaccine development. Vaccine 30: 142-154.

Ranasinghe C 2014. New advances in mucosal immunization. Immunol Lett doi: 10.1016/j.imlet.2014.01.006.

Rhee JH, Lee SE, Kim JY 2012. Mucosal vaccine adjuvants update. Clin Exp Vaccine Res 1: 50-63.

Sada-Ovalle I, Chiba A, Gonzales A, Brenner MB, Behar SM 2008. Innate invariant NKT cells recognize Mycobacterium tuberculosis-infected macrophages, produce interferon- $\gamma$ and kill intracellular bacteria. PLoS Pathog 4: e1000239.

Sato S, Kiyono H 2012. The mucosal immune system of the respiratory tract. Curr Opin Virol 2: 225-232.

Schreiber F, Huo Z, Giemza R, Woodrow M, Fenner N, Stevens Z, Dougan G, Prideaux S, Castello-Branco LRR, Lewis DJM 2010. An investigation of clinical and immunological events following repeated aerodigestive tract challenge infections with live $\mathrm{Myco-}$ bacterium bovis bacille Calmette-Guérin. Vaccine 28: 5427-5431. 
Sebina I, Cliff JM, Smith SG, Nogaro S, Webb EL, Riley EM, Dockrell HM, Elliott AM, Hafalla JC, Cose S 2012. Long-lived memory B-cell responses following BCG vaccination 2012. PLoS ONE 7: e51381.

Succi RCM 1985. BCG. In CK Farhat, Fundamentos e prática das imunizações em clínica médica e pediatria, Atheneu, Rio de Janeiro, p. 27-41.

Tameris MD, Mc Shane H, Mc Clain JB, Landry B, Lochhart S, Luabeya AK, Geldenhuys H, Shea J, Hussey G, van der Merwe L, de Kock M, Scriba T, Walker R, Hanekom W, Hatherill R, Mahomed H 2013. Lessons learnt from the first efficacy trial of a new infant tuberculosis vaccine since BCG. Tuberculosis (Edinb) 93: 143-149.

Thakur A, Pedersen LE, Jungersen G 2012. Immune markers and correlates of protection for vaccine induced immune responses. $\mathrm{Vac}$ cine 30: 4907-4920.

Valadas E 2004. Nontuberculous mycobacteria: clinical importance and relevance to bacille Calmette-Guérin vaccination. Clin Infect Dis 39: 457-458.
Vipond J, Cross ML, Lambeth MR, Clark S, Aldwell FE, Williams A 2008. Immunogenicity of orally-delivered lipid-formulated BCG vaccines and protection against Mycobacterium tuberculosis infection. Microbes Infect 10: 1577-1581.

Wang D, Xu J, Feng Y, Liu Y, Mchenga SS, Shan F, Sasaki J, Lu C 2010. Liposomal oral DNA vaccine (mycobacterium DNA) elicits immune response. Vaccine 28: 3134-3142.

Weiner 3rd J, Kaufmann SHE 2014. Recent advances towards tuberculosis control: vaccines and biomarkers. J Intern Med 275: 467-480.

WHO - World Health Organization 2013. Global tuberculosis report 2013. Available from: who.int/tb/publications/global_report/en/.

Woodrow KA, Bennett KM, Lo DD 2012. Mucosal vaccine design and delivery. Annu Rev Biomed Eng 14: 17-46.

Zaman M, Chandrudu S, Toth I 2013. Strategies for intranasal delivery of vaccines. Drug Deliv Transl Res 3: 100-109.

Zhang W, Zhang Y, Zheng H, Pan Y, Liu H, Du P, Wan L, Liu J, Zhu B, Zhao G, Chen C, Wan K 2013. Genome sequencing and analysis of BCG vaccine strains. PLoS ONE 8: e71243. 